\title{
EFFECTIVE LOWER BOUNDS FOR SOME LINEAR FORMS
}

\author{
BY
}

T. W. CUSICK

\begin{abstract}
It is proved that if $1, \alpha, \beta$ are numbers, linearly independent over the rationals, in a real cubic number field, then given any real number $d \geqslant 2$, for any integers $x_{0}, x_{1}, x_{2}$ such that $\left|\operatorname{norm}\left(x_{0}+\alpha x_{1}+\beta x_{2}\right)\right| \leqslant d$, there exist effectively computable numbers $c>0$ and $k>0$ depending only on $\alpha$ and $\beta$ such that $\left|x_{1} x_{2}\right|\left(\log \left|x_{1} x_{2}\right|\right)^{k \log d}\left|x_{0}+\alpha x_{1}+\beta x_{2}\right|>c$ holds whenever $x_{1} x_{2} \neq 0$. It would be of much interest to remove the dependence on $d$ in the exponent of $\log \left|x_{1} x_{2}\right|$, for then, among other things, one could deduce, for cubic irrationals, a stronger and effective form of Roth's Theorem.
\end{abstract}

1. Introduction. Let $\|x\|$ denote the nonnegative distance from $x$ to the nearest integer. The well-known theorem of Roth [11] asserts that for any real irrational algebraic number $\alpha$ and any $\epsilon>0$, there is a constant $c>0$ such that $q^{1+\epsilon}\|q \alpha\|>c$ holds for all integers $q>0$. Schmidt [12] has generalized this result to any number of dimensions. He proved that if $\alpha_{1}, \ldots, \alpha_{n}$ are any real algebraic numbers such that $1, \alpha_{1}, \ldots, \alpha_{n}$ are linearly independent over the rationals, then for any $\epsilon>0$ there is a constant $c_{n}>0$ such that

$$
\left|q_{1} q_{2} \cdots q_{n}\right|^{1+\epsilon}\left\|q_{1} \alpha_{1}+\cdots+q_{n} \alpha_{n}\right\|>c_{n}
$$

holds for all nonzero integers $q_{1}, \ldots, q_{n}$. He also proved a dual result, namely that, under the same hypotheses on $\alpha_{1}, \ldots, \alpha_{n}$, for any $\epsilon>0$ there is a constant $c_{n}^{\prime}>0$ such that

$$
q^{1+\epsilon}\left\|q \alpha_{1}\right\| \cdots\left\|q \alpha_{n}\right\|>c_{n}^{\prime}
$$

holds for all integers $q>0$.

The theorems of Roth and Schmidt are noneffective, that is, the constants $c, c_{n}, c_{n}^{\prime}$ cannot be effectively computed. Much recent work has been done on the problem of establishing effective lower bounds for $\left\|q_{1} \alpha_{1}+\cdots+q_{n} \alpha_{n}\right\|$, where $\alpha_{1}, \ldots, \alpha_{n}$ are algebraic numbers. All of the effective lower bounds obtained so far are appreciably weaker than (1) (see the survey articles by Baker [1], [3]).

The main result of this paper is:

Presented to the Society, November 1, 1975 ; received by the editors March 13, 1975. AMS (MOS) subject classifications (1970). Primary 10F10, 10F25; Secondary $10 \mathrm{F05}$.

Key words and phrases. Diophantine approximation, Roth's Theorem, real cubic fields, Baker's effective estimates. 
THEOREM 1. Suppose $1, \alpha$ and $\beta$ are numbers, linearly independent over the rationals, in a real cubic number field. Given any number $d \geqslant 2$, for any integers $x_{0}, x_{1}, x_{2}$ such that $\mid$ norm $\left(x_{0}+\alpha x_{1}+\beta x_{2}\right) \mid \leqslant d$, there exist effectively computable numbers $c>0$ and $k>0$ depending only on $\alpha$ and $\beta$ such that

$$
\left|x_{1} x_{2}\right|\left(\log \left|x_{1} x_{2}\right|\right)^{k \log d}\left|x_{0}+\alpha x_{1}+\beta x_{2}\right|>c
$$

holds whenever $\left|x_{1} x_{2}\right|>1$.

The hypotheses of Theorem 1 are very restrictive, but inequality (3) is both effective and stronger than (1). Indeed, (3) is nearly best possible, for it follows from some work of Peck [10] that if $1, \alpha, \beta$ are numbers, linearly independent over the rationals, in a real cubic number field, then the inequality

$$
\left|x_{1} x_{2}\right|\left(\log \left|x_{1} x_{2}\right|\right)^{1 / 2}\left|x_{0}+\alpha x_{1}+\beta x_{2}\right|<1
$$

has infinitely many solutions in integers $x_{0}, x_{1}, x_{2}$ with $x_{1} x_{2} \neq 0$. (Peck actually proved that $q \log q\|q \alpha\|\|q \beta\|<1$ has infinitely many solutions with $q>0$; one obtains the dual inequality stated above by using a transference principle of Mahler [9]. The argument, in a slightly different setting, is given in Cassels and Swinnerton-Dyer [6, p. 82].) Thus for fixed $d$ inequality (3) can only be improved by decreasing the exponent of $\log \left|x_{1} x_{2}\right|$.

Of course it is natural to hope that the factor $\log d$ in the exponent of $\log \left|x_{1} x_{2}\right|$ could be removed altogether, for then Theorem 1 would hold with no condition on the norm of $x_{0}+\alpha x_{1}+\beta x_{2}$. This hope is plausible from a metrical point of view, because it is known (see Gallagher [8] and Spencer [13]) that for almost all pairs of real numbers $\alpha, \beta$ an inequality of the form (3) holds with $k \log d$ replaced by an absolute constant. If Theorem 1 were strengthened in this way, then it would follow from an application of Mahler's transference principle that there exist effectively computable numbers $a>0$ and $c>0$ depending only on $\alpha$ and $\beta$ such that $q(\log q)^{a}\|q \alpha\|\|q \beta\|>c$ holds for all $q>0$. As an immediate corollary, we would have an inequality for all cubic irrationals which is both effective and stronger than Roth's Theorem.

2. Preliminaries. Let $F$ denote the real cubic field containing $\alpha$ and $\beta$. For any number $\gamma$ in $F$, we let $\gamma^{(0)}=\gamma, \gamma^{(1)}=\gamma^{\prime}, \gamma^{(2)}=\gamma^{\prime \prime}$ denote the conjugates of $\gamma$. Let $G$ denote the smallest field containing $\alpha, \alpha^{\prime}, \alpha^{\prime \prime}, \beta, \beta^{\prime}$ and $\beta^{\prime \prime}$. If $F$ is a cyclic (totally real) cubic field, then $F=G$; otherwise $G$ is a normal field of degree 6 , which is totally real if and only if $F$ is.

From here until $\S 5$ we suppose that $F$ is totally real. The case of nontotally real $F(\S 5)$ turns out to be simpler.

Let $M$ denote the module $\left\{x_{0}+\alpha x_{1}+\beta x_{2}: x_{0}, x_{1}, x_{2}\right.$ rational integers $\}$ and let $T=\{\gamma: \gamma$ in $F, M$ contains $\gamma M\}$ denote the coefficient ring of $M$. It follows from Dirichlet's unit theorem [4, p. 112, Theorem 5] that there exist two 
units $\theta_{1}$ and $\theta_{2}$ in $T$ such that every unit in $T$ has the form $\pm \theta_{1}^{m} \theta_{2}^{n}$, where $m$ and $n$ are rational integers.

Let

$$
\mathbf{R}=\operatorname{det}\left[\begin{array}{ll}
\log \left|\theta_{1}^{\prime}\right| & \log \left|\theta_{2}^{\prime}\right| \\
\log \left|\theta_{1}^{\prime \prime}\right| & \log \left|\theta_{2}^{\prime \prime}\right|
\end{array}\right] .
$$

The regulator of $T$ is $|R|$, so $|R|$ is not zero and is independent of the choice of the fundamental units $\theta_{1}, \theta_{2}$ for $T[4, \mathrm{p} .115]$.

If $\theta_{1}, \theta_{2}$ is a pair of fundamental units for $T$, then so is $\theta_{1}^{p} \theta_{2}^{q}, \theta_{1}^{r} \theta_{2}^{s}$ for integers $p, q, r, s$ such that $p s-q r= \pm 1$. Hence for any $\epsilon_{0}>0$ (however small) and any $L>0$ (however large) it is possible to choose $\theta_{1}$ in the pair $\theta_{1}, \theta_{2}$ of fundamental units so that

$$
|| \theta_{1} / \theta_{1}^{\prime \prime}|-1|<\epsilon_{0} \text { and }\left|\theta_{1}^{\prime}\right|>L .
$$

Later on in the paper (Lemmas 5 and 6) we shall require the inequalities (5) for certain explicitly calculable $\epsilon_{0}$ and $L$. We assume from now on that a fixed pair $\theta_{1}, \theta_{2}$ of fundamental units for $T$ has been chosen in such a way that (5) holds for the relevant $\epsilon_{0}$ and $L$. It is convenient to assume further that $\theta_{1}, \theta_{2}$ have been chosen in such a way that $\theta_{1}$ and $\theta_{2}$ both have norm +1 and

$$
\mathbf{R}=\text { regulator of } T>0
$$

(of course given any pair $\theta_{1}, \theta_{2}$ we can satisfy (6) by simply replacing $\theta_{2}$ by $\theta_{2}^{-1}$, if necessary).

Define

$$
D(1)=-\log \left|\theta_{2}^{\prime}\right|+\log \left|\theta_{2}^{\prime \prime}\right|, \quad D(2)=\log \left|\theta_{1}^{\prime}\right|-\log \left|\theta_{1}^{\prime \prime}\right| .
$$

Neither $D(1)$ nor $D(2)$ is zero. (Of course, $D(2)>0$ by (5) if $L$ is large enough; we assume this is the case.) Indeed, more generally, if $\gamma \neq \pm 1$ is any element of $F$, then $\gamma \gamma^{\prime 2} \neq \pm 1$. For $\gamma \gamma^{\prime 2}= \pm 1$ implies $\gamma^{\prime} \gamma^{\prime \prime 2}=\gamma^{\prime \prime} \gamma^{2}= \pm 1$, and solving these equations gives $\gamma^{9}= \pm 1$, which implies $\gamma= \pm 1$. We define $\eta_{0}, \eta_{1}, \eta_{2}$ (numbers which will be encountered later on) as follows:

$$
\eta_{i}=\left|\theta_{1}^{(i)}\right|^{D(1)}\left|\theta_{2}^{(i)}\right|^{D(2)} \quad(0 \leqslant i \leqslant 2) .
$$

It is easy to see that

$$
\log \eta_{0}=-2 \mathbf{R}, \quad \log \eta_{1}=\log \eta_{2}=\mathbf{R} .
$$

For any pair of rational integers $m, n$ define

$$
R(m, n)=\theta_{1}^{m} \theta_{2}^{n}
$$

Since 1 is in $M$, the numbers $R(m, n)$ are in $M$ for all $m, n$ and we have

$$
R(m, n)=a_{0}(m, n)+a_{1}(m, n) \alpha+a_{2}(m, n) \beta
$$


for some rational integers $a_{i}(m, n)(0 \leqslant i \leqslant 2)$.

We now need to consider some results about the solutions in integers $x_{0}$, $x_{1}, x_{2}$ of the inequality

$$
\left|x_{0}+\alpha x_{1}+\beta x_{2}\right| \max \left(x_{1}^{2}, x_{2}^{2}\right)<Q,
$$

where $Q$ is any constant $\geqslant 1$. Later on we will use these results in dealing with the expression $\left|x_{0}+\alpha x_{1}+\beta x_{2}\right|\left|x_{1} x_{2}\right|$ in the proof of Theorem 1 .

We first recall the well-known result $[5, \mathrm{p} .79]$ that for $1, \alpha, \beta$ linearly independent in a real cubic field there is a constant $k$ satisfying $0<k<1$ and depending only on $\alpha$ and $\beta$ such that

$$
\kappa \leqslant\left|x_{0}+\alpha x_{1}+\beta x_{2}\right| \max \left(x_{1}^{2}, x_{2}^{2}\right)
$$

holds for all integers $x_{0}, x_{1}, x_{2}$ with $x_{1}$ and $x_{2}$ not both zero.

Next we need the following lemma.

LEMMA 1. Let $a$ be any nonzero rational number. Then in the module $M$ there is a finite (possibly empty) set of numbers $\mu_{1}, \ldots, \mu_{k}$ with norm a such that every solution $\gamma$ of norm $\gamma=a, \gamma$ in $M$, has a unique representation in the form $\gamma=\mu_{i} R(m, n)$ for some $i(1 \leqslant i \leqslant k)$ and some $m, n$.

Proof. See [4, p. 118, Theorem 1].

From now on we use $c_{1}, c_{2}, \ldots$ to denote positive constants which depend at most on $\alpha, \beta, \theta_{1}$ and $\theta_{2}$. Of course, since we have assumed that $\theta_{1}$ and $\theta_{2}$ have been chosen, we could as well say that $c_{1}, c_{2}, \ldots$ depend at most on $\alpha$ and $\beta$. However, we shall sometimes wish to know that certain constants are the same whatever choice of $\theta_{1}$ and $\theta_{2}$ might have been made; this is not in general true for $c_{1}, c_{2}, \ldots$.

If we let $\xi=x_{0}+\alpha x_{1}+\beta x_{2}$ and $\xi^{(i)}=x_{0}+\alpha^{(i)} x_{1}+\beta^{(i)} x_{2} \quad(0 \leqslant i \leqslant 2)$, then clearly

$$
\max _{0<i<2}\left|\xi^{(i)}\right| \leqslant c_{1} \max _{0<i \leqslant 2}\left|x_{i}\right|
$$

Since in Theorem 1 we are concerned with a lower bound for $|\xi|$, we may clearly assume that $x_{0}$ is always taken to be the nearest integer to $\alpha x_{1}+\beta x_{2}$. Thus $|\xi| \leqslant 1 / 2$ holds and so

$$
\max _{0<i<2}\left|x_{i}\right| \leqslant c_{2} \max \left(\left|x_{1}\right|,\left|x_{2}\right|\right) \text {. }
$$

It follows from (12) and (13) that if (10) holds, then

$$
|\xi| \max \left(\left.i \xi^{\prime}\right|^{2},\left|\xi^{\prime \prime}\right|^{2}\right)<Q\left(c_{1} c_{2}\right)^{2} \text {. }
$$

By (14), norm $\xi$ is bounded if $\xi$ satisfies (10), so by Lemma 1 in looking at (10) we need only consider solutions of

$$
|\delta R(m, n)| \max \left(b_{1}(m, n)^{2}, b_{2}(m, n)^{2}\right)<Q
$$


where

$$
\xi=\delta R(m, n)=b_{0}(m, n)+b_{1}(m, n) \alpha+b_{2}(m, n) \beta
$$

for some rational integers $b_{i}(m, n)(0 \leqslant i \leqslant 2)$ and where $\delta$ runs through some finite set (depending on $Q$ ) in $M$. Call this set $\Delta(Q)$.

We can be more explicit about the membership of $\Delta(Q)$. Let

$$
\tau=\min \{\mid \text { norm } \gamma \mid: \gamma \neq 0 \text { in } M\} .
$$

Of course the set on the right-hand side is discrete, and $\tau>0$. It now follows from (14) that if $|\xi| \leqslant 1 / 2$ and (10) holds, then

$$
\tau \leqslant \mid \text { norm } \delta \mid<Q\left(c_{1} c_{2}\right)^{2} \text {. }
$$

Thus by Lemma 1 , for each of the finite number of values $d$ of norm $\delta$ satisfying (18), $\Delta(Q)$ contains some finite number of elements $\mu_{1}^{[d]}, \ldots, \mu_{k(d)}^{[d]}$, all of norm $d$.

We use the notation $\left(a_{i j}\right)$ for a matrix with entry $a_{i j}$ in the $i$ th row and $j$ th column.

LEMMA 2. The integers $a_{i}(m, n)(0 \leqslant i \leqslant 2)$ in (9) satisfy

$$
a_{i}(m, n)=\sum_{j=0}^{2} a_{i j} R^{(j)}(m, n) \quad(0 \leqslant i \leqslant 2)
$$

where the coefficient matrix $A=\left(a_{i j}\right)(0 \leqslant i, j \leqslant 2)$ satisfies

$$
A=\left[\begin{array}{lll}
1-\alpha a_{10}-\beta a_{20} & 1-\alpha^{\prime} a_{11}-\beta^{\prime} a_{21} & 1-\alpha^{\prime \prime} a_{12}-\beta^{\prime \prime} a_{22} \\
D_{M}^{-1}\left(\beta^{\prime}-\beta^{\prime \prime}\right) & D_{M}^{-1}\left(\beta^{\prime \prime}-\beta\right) & D_{M}^{-1}\left(\beta-\beta^{\prime}\right) \\
D_{M}^{-1}\left(\alpha^{\prime \prime}-\alpha^{\prime}\right) & D_{M}^{-1}\left(\alpha-\alpha^{\prime \prime}\right) & D_{M}^{-1}\left(\alpha^{\prime}-\alpha\right)
\end{array}\right]
$$

here

$$
D_{M}=\operatorname{det}\left[\begin{array}{ll}
\alpha^{\prime}-\alpha & \beta^{\prime}-\beta \\
\alpha^{\prime \prime}-\alpha & \beta^{\prime \prime}-\beta
\end{array}\right]
$$

is one of the square roots of the discriminant of the module $M$.

The integers $b_{i}(m, n)(0 \leqslant i \leqslant 2)$ in (16) satisfy

$$
b_{i}(m, n)=\sum_{j=0}^{2} b_{i j} R^{(j)}(m, n) \quad(0 \leqslant i \leqslant 2)
$$

where the coefficient matrix $B=\left(b_{i j}\right)(0 \leqslant i, j \leqslant 2)$ is given by

$$
B=\left(\delta^{(j)} a_{i j}\right) \quad(0 \leqslant i, j \leqslant 2) .
$$

Proof. Let $C$ denote the matrix on the right-hand side of (21). We have the identity 


$$
C\left[\begin{array}{l}
a_{1}(m, n) \\
a_{2}(m, n)
\end{array}\right]=\left[\begin{array}{l}
R^{\prime}(m, n)-R(m, n) \\
R^{\prime \prime}(m, n)-R(m, n)
\end{array}\right] .
$$

Multiplying both sides by the inverse matrix $C^{-1}$ gives (19) and (20) (the case $i=0$ of (19) follows at once from the cases $i=1,2$ and (9)).

To prove (22) and (23) we apply the argument above to the identity

$$
C\left[\begin{array}{l}
b_{1}(m, n) \\
b_{2}(m, n)
\end{array}\right]=\left[\begin{array}{l}
\delta^{\prime} R^{\prime}(m, n)-\delta R(m, n) \\
\delta^{\prime \prime} R^{\prime \prime}(m, n)-\delta R(m, n)
\end{array}\right] .
$$

We note that any ratio of two numbers in the same row or column of $A$ or $B$ is in the field $G$. If $F$ is cyclic (so $F=G$ ), then $D_{M}$ is a rational integer and $a_{i 1}=a_{i 0}^{\prime}, a_{i 2}=a_{i 0}^{\prime \prime}(0 \leqslant i \leqslant 2)$. In this case all the entries in $A$ and $B$ are themselves in $F$.

3. A technical lemma. It is clear from Lemma 1 that in $\Delta(Q)$ defined above we may replace any of the elements $\mu_{i}^{[d]}$ of norm $d$ by $R(m, n) \mu_{i}^{[d]}$, where $R(m, n)$ is any unit in the coefficient ring $T$. Let $\Delta(Q, d)$ denote the subset of $\Delta(Q)$ made up of those numbers with norm $\leqslant d$. It follows from (18) that $\Delta(Q, d)$ has a fixed number of elements for all $Q>d /\left(c_{1} c_{2}\right)^{2}$. Thus we may suppose $\Delta(Q, d)$ is independent of $Q$ for $Q$ large enough; call this set $\Delta^{*}(d)$. In the proof of Theorem 1 it will be necessary to ensure that for any $\mu$ in $\Delta^{*}(d)$, the absolute value of the ratio of any two conjugates of $\mu$ in $F$ is bounded by a constant independent of the choice of $\theta_{1}, \theta_{2}$. This can be achieved by replacing each element $\mu$ of $\Delta^{*}(d)$ by a suitable $R(m, n) \mu$; the following lemma is needed to show that such a replacement is always possible.

LEMma 3. Let $\mu$ be any element of $M$. Then there exists a number $\zeta$ in $M$ such that $\zeta / \mu$ is a unit in $T$ and

$$
\max _{0<i, j \leqslant 2}\left|\zeta^{(i)} / \zeta^{(j)}\right| \leqslant c_{3}
$$

where $c_{3}$ is a constant depending only on the module $M$.

Proof. Let $\varphi_{1}, \varphi_{2}$ be any pair of fundamental units for $T$. If we define $\zeta=\varphi_{1}^{x} \varphi_{2}^{y} \mu$, then we can choose $x$ and $y$ so that

$$
\left|\zeta^{(i)}\right| \leqslant c \mid \text { norm }\left.\zeta\right|^{1 / 3} \quad(0 \leqslant i \leqslant 2),
$$

where $c$ is a constant depending only on $M[4, \mathrm{pp} .122-123]$. Then $\zeta / \mu$ is a unit in $T$ and clearly (24) holds.

Given any number $\mu$ in $\Delta^{*}(d)$, we can replace $\mu$ by the corresponding number $\zeta$ given in Lemma 3. Thus we can assume

$$
\text { for any } \mu \text { in } \Delta^{*}(d), \max _{0<i, j \leqslant 2}\left|\mu^{(i)} / \mu^{(j)}\right| \leqslant c_{3},
$$


where $c_{3}$ depends only on $\alpha$ and $\beta$, and not on the choice of $\theta_{1}, \theta_{2}$ in $T$. Furthermore, if $\mu_{1}$ is in $\Delta^{*}\left(d_{1}\right)$ and, for some $d_{2} \geqslant d_{1}, \mu_{2}$ is a number in $\Delta^{*}\left(d_{2}\right)$ such that $\mu_{2} / \mu_{1}=(p / q) R(m, n)$ for some unit $R(m, n)$ in $T$, where $p, q$ are rational integers and $p / q \geqslant 1$, then we can assume that $\mu_{2} / \mu_{1}=p / q$. We shall say that each number $\mu$ in $\Delta^{*}(d)$ is canonical if this last assumption and (25) both hold.

We suppose from now on that every element of $\Delta^{*}(d)$ is canonical.

4. Proof of Theorem 1, totally real case. The first major ingredient in the proof of Theorem 1 is the following theorem of Baker $[2, I]$.

THEOREM 2 (BAKER). Let $\sigma_{1}, \ldots, \sigma_{n}$ be nonzero algebraic numbers with degrees at most $\Sigma$ and let the heights of $\sigma_{1}, \ldots, \sigma_{n-1}$ and $\sigma_{n}$ be at most $A^{\prime}$ and $A(\geqslant 2)$, respectively. Then for some effectively computable number $C>0$ depending only on $n, \Sigma$ and $A^{\prime}$, the inequalities

$$
0<\left|p_{1} \log \sigma_{1}+\cdots+p_{n} \log \sigma_{n}\right|<C^{-\log A \log P}
$$

have no solution in rational integers $p_{1}, \ldots, p_{n}$ with absolute value at most $P(\geqslant 2)$.

We have stated the theorem in the general form given by Baker, though we require only the special case $n=3, p_{3}=-1$ for the following lemma. We do not need the further refinement of Theorem 2 in the case $p_{n}=-1$ which is given by Baker [2, II, Theorem 2].

LemmA 4. Given any number $d \geqslant 2$, if $\delta$ is canonical, $x_{1} x_{2} \neq 0$ and $\xi=$ $\delta R(m, n)=x_{0}+\alpha x_{1}+\beta x_{2}$ satisfies |norm $\xi \mid \leqslant d$, then

$$
\left|x_{1} x_{2}\left(x_{0}+\alpha x_{1}+\beta x_{2}\right)\right| \geqslant c_{4} /(\max (|m|,|n|))^{c_{5} \log d} \text {. }
$$

Proof. We may assume that $\left|x_{1}\right| \geqslant\left|x_{2}\right|$; an argument parallel to what follows takes care of the case $\left|x_{1}\right|<\left|x_{2}\right|$. Since

$$
\left|x_{1} x_{2} \xi\right|=\left|x_{2} / x_{1}\right| x_{1}^{2}|\xi|=\left|x_{2} / x_{1}\right||\xi| \max \left(x_{1}^{2}, x_{2}^{2}\right)
$$

it follows from (11) that the lemma is true if we can prove

$$
\left|x_{2} / x_{1}\right|>c_{6} /(\max (|m|,|n|))^{c_{5} \log d} \text {. }
$$

To do this, we first observe that by (16) and Lemma 2

$$
x_{1}=a_{10} \xi+a_{11} \xi^{\prime}+a_{12} \xi^{\prime \prime}, \quad x_{2}=a_{20} \xi+a_{21} \xi^{\prime}+a_{22} \xi^{\prime \prime} .
$$

We may assume that $|\xi|$ is very small, for otherwise (26) is trivial. If $|\xi|$ is small enough, it follows that

$$
\left|x_{2}\right| \geqslant 1 / 2\left|a_{21} \xi^{\prime}+a_{22} \xi^{\prime \prime}\right| \text { and }\left|x_{1}\right| \leqslant c_{7} \max \left(\left|\xi^{\prime}\right|,\left|\xi^{\prime \prime}\right|\right) \text {, }
$$




$$
\left|x_{2} / x_{1}\right| \geqslant c_{8}\left|a_{21} \xi^{\prime}+a_{22} \xi^{\prime \prime}\right| / \max \left(\left|\xi^{\prime}\right|,\left|\xi^{\prime \prime}\right|\right) \text {. }
$$

We may assume $\left|\xi^{\prime \prime}\right| \geqslant\left|\xi^{\prime}\right|$; a similar argument takes care of the case $\left|\xi^{\prime \prime}\right|<$ $\left|\xi^{\prime}\right|$. Thus we need a lower bound for the absolute value of

$$
a_{21}\left(\xi^{\prime} / \xi^{\prime \prime}\right)+a_{22}=a_{22}\left(\left(b_{21} \theta_{1}^{\prime m} \theta_{2}^{\prime n} / b_{22} \theta_{1}^{\prime \prime m} \theta_{2}^{\prime n}\right)+1\right) \text {. }
$$

Applying Theorem 2 with $n=3, \sigma_{1}=\left|\theta_{1}^{\prime} / \theta_{1}^{\prime \prime}\right|, \sigma_{2}=\left|\theta_{2}^{\prime} / \theta_{2}^{\prime \prime}\right|, \sigma_{3}=\left|b_{22} / b_{21}\right|$, $p_{3}=-1$, we find that for any integers $m, n$ not both zero either

or

$$
\left|b_{21} \theta_{1}^{\prime m} \theta_{2}^{\prime n}\right|=\left|b_{22} \theta_{1}^{\prime \prime m} \theta_{2}^{\prime \prime n}\right|
$$

$$
|m \log | \theta_{1}^{\prime} / \theta_{1}^{\prime \prime}|+n \log | \theta_{2}^{\prime}\left|\theta_{2}^{\prime \prime}\right|-\log \left|b_{22} / b_{21}\right| \mid \geqslant 1 /(\max (|m|,|n|))^{\nu},
$$

where $\nu>0$ is a constant depending on the degree and height of $b_{22} / b_{21}$. In fact, Theorem 2 shows that $\nu$ is of the form $k_{1} \log \left(\right.$ height $\left.\left(b_{22} / b_{21}\right)\right)$ where $k_{1}$ depends on the degree but not the height of $b_{22} / b_{21}$. However, $b_{22} / b_{21}=$ $\delta^{\prime \prime}\left(\alpha^{\prime}-\alpha\right) / \delta^{\prime}\left(\alpha-\alpha^{\prime \prime}\right)$ is a number in $G$. Since $\delta$ is canonical, it follows from (25) with $\mu=\delta$ that the heights of $\delta^{\prime}$ and $\delta^{\prime \prime}$ are less than $k_{2} d$, where $k_{2}$ depends only on $\alpha$ and $\beta$. This implies (after some easy estimates) that the height of $b_{22} / b_{21}$ is less than $k_{3} d^{\Gamma}$, where $k_{3}$ depends only on $\alpha$ and $\beta$, and $\Gamma$ is an absolute constant. Hence in (31) we can take $\nu=c_{9} \log d$. Now (27) follows easily after exponentiating (31) and applying the inequality $e^{x}>1+x$.

It remains to consider the case when (30) holds, so Theorem 2 does not apply. If (30) is true with the absolute value signs removed, then $a_{21}\left(\xi^{\prime} / \xi^{\prime \prime}\right)+$ $a_{22}=2 a_{22}$, so (27) is trivially true. If, on the other hand, $a_{21} \xi^{\prime}+a_{22} \xi^{\prime \prime}=0$, then for some integers $r$ and $s$ we have (using (20))

$$
-\frac{b_{22}}{b_{21}}=\frac{\delta^{\prime \prime}\left(\alpha-\alpha^{\prime}\right)}{\delta^{\prime}\left(\alpha-\alpha^{\prime \prime}\right)}=\left(\frac{\theta_{1}^{\prime}}{\theta_{1}^{\prime \prime}}\right)^{r}\left(\frac{\theta_{2}^{\prime}}{\theta_{2}^{\prime \prime}}\right)^{s} \text {. }
$$

Suppose (32) holds for two different canonical values of $\delta$, say $\delta_{1}$ and $\delta_{2}$ (with possibly different values of $r$ and $s$, of course). It follows that for some integers $t$ and $u$ we have

$$
\left(\delta_{1}^{\prime \prime} / \delta_{2}^{\prime \prime}\right) \theta_{1}^{\prime \prime t} \theta_{2}^{\prime \prime u}=\left(\delta_{1}^{\prime} / \delta_{2}^{\prime}\right) \theta_{1}^{\prime t} \theta_{2}^{\prime u} .
$$

The two sides of this equality are numbers in different conjugate fields of $F$; hence both numbers are equal to some rational number $q / p$. This means $\delta_{1}(p / q) \theta_{1}^{t} \theta_{2}^{u}=\delta_{2}$, but since $\delta_{1}$ and $\delta_{2}$ are canonical we must have $t=u=0$ (see $\S 3$ ). Therefore even if (32) holds for various values of $\delta$, the integers $r$ and $s$ do not change. It follows that

$$
b_{21} \theta_{1}^{\prime m} \theta_{2}^{\prime n}+b_{22} \theta_{1}^{\prime \prime m} \theta_{2}^{\prime \prime n}=0
$$

is possible for at most one integer pair $m, n$. Even if there is such an exceptional 
pair $m, n$, we can still ensure that (27) holds by adjusting the constant $c_{6}$, if necessary. This completes the proof of the lemma.

In view of Lemma 4, if we could prove that

$$
\log \left|x_{1} x_{2}\right|>c \max (|m|,|n|)
$$

holds for some constant $c$ depending only on $\alpha$ and $\beta$, then Theorem 1 would be proved. An inequality of the form (33) follows easily from (28) and the fact that $\xi=\delta \theta_{1}^{m} \theta_{2}^{n}$. However, the constant $c$ obtained in this way depends on $\delta$ (and so on $d$ ); with such a constant, inequality (33) is useless for proving Theorem 1. In order to get around this difficulty, we need more information about $m$ and $n$. The following theorem, which is the second major ingredient in the proof of Theorem 1, gives us what we need.

THEOREM 3. Suppose there exists $\xi=x_{0}+\alpha x_{1}+\beta x_{2}$ with norm of absolute value $w$ such that (15) and (16) hold, with $\delta$ canonical. Then $|m-\lambda n| \leqslant K$ where $\lambda=D(1) / D(2)$ and $K$ is a constant depending only on $Q, w, \alpha, \beta$, and $\theta_{1}$. We can take

$$
K=\frac{\log (h Q / w)}{D(2)}=\frac{\log (h Q / w)}{\log \left|\theta_{1}^{\prime} / \theta_{1}^{\prime \prime}\right|}
$$

where $h$ depends only on $\alpha$ and $\beta$, and not on the choice of $\theta_{1}$ and $\theta_{2}$.

Proof. This is essentially a special case of the theorem of Cusick [7, p. 19], except for the evaluation of $K$. I repeat the proof of [7] with the changes needed to get an explicit $K$.

The norm $\xi \xi^{\prime} \xi$ ' has absolute value $w$, so by (14)

$$
|\xi| \min _{i=1,2}\left|\xi^{(i)}\right|^{2}=w^{2}\left(|\xi| \max _{i=1,2}\left|\xi^{(i)}\right|^{2}\right)^{-1}>\left(w / c_{1} c_{2}\right)^{2} Q^{-1} .
$$

Combining (14) and (34) gives

$$
\max _{i=1,2}\left|\xi^{(i)}\right|<\left(c_{1} c_{2}\right)^{2} Q w^{-1} \min _{i=1,2}\left|\xi^{(i)}\right|
$$

for any $\xi=\delta R(m, n)$ which satisfies (15).

If (15) holds then (25) with $\mu=\delta$ and (35) give

it follows that

$$
\max _{i=1,2}\left|R^{(i)}(m, n)\right|<\left(c_{1} c_{2}\right)^{2} c_{3} Q w^{-1} \min _{i=1,2}\left|R^{(i)}(m, n)\right| ;
$$

$$
|\log | R^{\prime}(m, n)|-\log | R^{\prime \prime}(m, n)||<\log \left(\left(c_{1} c_{2}\right)^{2} c_{3} Q / w\right),
$$

and so

$$
\begin{aligned}
& m \log \left|\theta_{1}^{\prime}\right|+n \log \left|\theta_{2}^{\prime}\right|=\log \left|R^{\prime \prime}(m, n)\right|+\epsilon, \\
& m \log \left|\theta_{1}^{\prime \prime}\right|+n \log \left|\theta_{2}^{\prime \prime}\right|=\log \left|R^{\prime \prime}(m, n)\right|,
\end{aligned}
$$

where $|\epsilon|<\log \left(\left(c_{1} c_{2}\right)^{2} c_{3} Q / w\right)$. 
The system (36) has for its determinant the regulator $\mathbf{R}$ given by (4). Thus solving for $m$ and $n$ gives

Hence

$$
\begin{gathered}
m=D(1) \mathbf{R}^{-1} \log \left|R^{\prime \prime}(m, n)\right|+\epsilon \mathbf{R}^{-1} \log \left|\theta_{2}^{\prime \prime}\right|, \\
n=D(2) \mathbf{R}^{-1} \log \left|R^{\prime \prime}(m, n)\right|-\epsilon \mathbf{R}^{-1} \log \left|\theta_{1}^{\prime \prime}\right| .
\end{gathered}
$$

$$
|m-\lambda n|=\left|\epsilon \mathbf{R}^{-1}\left(\log \eta_{2}\right) D(2)^{-1}\right| \leqslant D(2)^{-1} \log \left(\left(c_{1} c_{2}\right)^{2} c_{3} Q / w\right)
$$

where $\eta_{2}$ is defined by (7), and for the inequality we have used (8) and the upper bound on $|\epsilon|$. It is evident that $c_{1}, c_{2}$ and $c_{3}$ depend only on $\alpha$ and $\beta$, so we have Theorem 3 with $h=\left(c_{1} c_{2}\right)^{2} c_{3}$.

As was remarked earlier, we obviously need to consider only those solutions of (10) where $x_{0}$ is the nearest integer to $\alpha x_{1}+\beta x_{2}$. Assuming this, we divide the integer solutions to (10) into classes according to the size of the left-hand side. In the first class the left-hand side of (10) is $\geqslant k$ (defined in (11)) and $<1$; in the second class the left side of $(10)$ is $\geqslant 1$ and $<2$; and in the $N$ th class $(N=2,3, \ldots)$ the left side of $(10)$ is $\geqslant 2^{N-2}$ and $<2^{N-1}$. Thus any integer pair $\left(x_{1}, x_{2}\right) \neq(0,0)$ is in one of the classes, since it will satisfy $(10)$ for $Q$ large enough. Using this division into classes and Theorem 3, we prove the following lemma, which gives inequality (33) with a suitable constant when $n>0$.

LEMMA 5. If $\xi=\delta R(m, n)=x_{0}+\alpha x_{1}+\beta x_{2}$ with $\delta$ canonical, $n>0$ and $x_{1} x_{2} \neq 0$, then $\log \left|x_{1} x_{2}\right|>c_{10} n$.

Proof. As in the proof of Lemma 4, we may assume $\left|x_{1}\right| \geqslant\left|x_{2}\right|$. Suppose $x_{1}, x_{2}$ is in the $N$ th class of solutions to (10), so (10) holds for some $Q<2^{N-1}$; also suppose $\mid$ norm $\delta \mid=w$. By Theorem 3 and (7), (8)

$$
|R(m, n)|=\exp \left(-2 R D(2)^{-1} n\right)\left|\theta_{1}\right|^{\epsilon}, \quad|\epsilon| \leqslant K .
$$

Since $\theta_{1} \theta_{1}^{\prime} \theta_{1}^{\prime \prime}=1$ and, if $\epsilon_{0}$ in (5) is chosen small enough and $L$ in $(5)$ is chosen large enough, $\left|\theta_{1}^{\prime \prime}\right| \approx\left|\theta_{1}\right|<1$, we have $2 \log \left|\theta_{1}\right|+\log \left|\theta_{1}^{\prime}\right| \approx 0$ and (using the definition of $K$ in the first inequality, the definition $D(2)=\log \left|\theta_{1}^{\prime}\right| \theta_{1}^{\prime \prime} \mid$ in the second inequality, and $Q<2^{N-1}$ in the third inequality)

$$
\begin{aligned}
\left|\theta_{1}\right|^{\epsilon} & \leqslant \exp \left(-\log (h Q / w) D(2)^{-1} \log \left|\theta_{1}\right|\right) \\
& \leqslant c_{11} \exp \left(-|\log | \theta_{1}|/ \log | \theta_{1} \theta_{1}^{\prime 2}|| \log Q\right) \\
& \leqslant c_{12} \exp \left(-N\left(1 / 3+\epsilon_{1}\right) \log 2\right),
\end{aligned}
$$

where $\epsilon_{1}>0$ is a small number depending on $\epsilon_{0}$ in (5) $\left(\epsilon_{1}\right.$ decreases if $\epsilon_{0}$ is chosen smaller).

By (18), $\left|\delta \delta^{\prime} \delta^{\prime \prime}\right|<Q\left(c_{1} c_{2}\right)^{2}$, so it follows from (25) and the fact that $\delta$ is canonical that

$$
|\delta| \leqslant c_{13} Q^{1 / 3} \leqslant c_{14} \exp ((N / 3) \log 2)
$$


Since $x_{1}, x_{2}$ is in the $N$ th class of solutions, we have

$$
2^{N-2} \leqslant i \delta R(m, n) \mid x_{1}^{2}
$$

if $N>1$ (for $N=1$, the left side of the above inequality should be the number $\kappa$ defined in (11)). Applying (37), (38) and (39) to (40), we obtain

$$
\begin{aligned}
x_{1}^{2} & \geqslant c_{15} 2^{N} \exp \left(-N\left(2 / 3+\epsilon_{1}\right) \log 2+2 \mathrm{R} D(2)^{-1} n\right) \\
& >c_{16} \exp \left(2 \mathrm{R} D(2)^{-1} n\right),
\end{aligned}
$$

where the second inequality holds if $\epsilon_{1}$ is small enough. The lemma follows at once.

The inequality of Theorem 1 follows from Lemmas 4 and 5 provided $n>0$. If $n \leqslant 0$, then a result stronger than Theorem 1 is true, as we shall show using the following lemma.

LEMma 6. Suppose $\xi=\delta R(m, n)=x_{0}+\alpha x_{1}+\beta x_{2}$ satisfies (10) with $\delta$ canonical and $x_{1}, x_{2}$ is in the jth class of solutions, $j \leqslant N$. If $n \leqslant N$ and $\left|x_{1}\right| \geq\left|x_{2}\right|>0$, then

$$
\left|x_{2} / x_{1}\right|>c_{17} / 2^{N} \text {. }
$$

Proof. Since $x_{1}, x_{2}$ is in the $N$ th class of solutions, we have

$$
|\delta R(m, n)| x_{1}^{2}<2^{N-1} \text {. }
$$

Since $\delta$ is canonical and by (18) has norm of absolute value at least $\tau,(25) \mathrm{im}$ plies

$$
|\delta| \geqslant c_{18}|\operatorname{norm} \delta|^{1 / 3} \geqslant c_{19} .
$$

Equality (37) holds as before, and by the same reasoning used to prove (38) (the only difference is that here we want a lower bound) we obtain

$$
\left|\theta_{1}\right|^{\epsilon} \geqslant c_{20} \exp \left(-N\left(1 / 3+\epsilon_{1}\right) \log 2\right) \text {. }
$$

Since $D(2)=\log \left|\theta_{1}^{\prime}\right| \theta_{1}^{\prime \prime} \mid$ is large if $L$ in $(5)$ is chosen large, we have

$$
\exp \left(-2 R D(2)^{-1} n\right) \geqslant \exp \left(-2 R D(2)^{-1} N\right) \geqslant \exp \left(-\epsilon_{2} N \log 2\right)
$$

where $\epsilon_{2}>0$ is a small number depending on $L$ in (5) $\left(\epsilon_{2}\right.$ decreases if $D(2)^{-1}$ is chosen smaller, that is, if $L$ is chosen larger). Using this inequality, (37), (42) and (43), we see that (41) gives

$$
x_{1}^{2}<c_{21} \exp \left(\left((4 / 3)+\epsilon_{1}+\epsilon_{2}\right) N \log 2\right) .
$$

If $\epsilon_{1}$ and $\epsilon_{2}$ are small enough and $x_{1} x_{2} \neq 0$, this easily gives the inequality of the lemma.

Now suppose $n \leqslant 0$ and the hypotheses of Lemma 6 hold (of course there is no loss of generality in assuming the hypothesis $\left.\left|x_{1}\right| \geqslant\left|x_{2}\right|\right)$. Since $x_{1}, x_{2}$ is in the $N$ th class of solutions, we have 


$$
\left|x_{1} x_{2} \xi\right|=\left|x_{2} / x_{1}\right| x_{1}^{2}|\xi| \geqslant c_{22}\left|x_{2} / x_{1}\right| 2^{N} \text {. }
$$

It follows from this and Lemma 6 that the inequality of Theorem 1 holds even without the logarithm factor, when $n \leqslant 0$.

It is, of course, not surprising that this stronger result holds in the cases $n \leqslant 0$ because of (37) and the fact that we need only consider those pairs $m, n$ for which $|\delta R(m, n)| \leqslant 1 / 2$. Since $R D(2)^{-1}>0$, this means that for a given $\delta$ only a finite number of $n \leqslant 0$ come into consideration.

5. Proof of Theorem 1, nontotally real case. In this section we suppose that $F$ is nontotally real. The proof of Theorem 1 in this case follows the general pattern of the proof for the totally real case ( $\$ \S 2$ to 4$)$, but the details are much simpler. Therefore we only sketch the proof.

The coefficient ring $T$ of the module $M$ has only one fundamental unit, say $\theta$, which we suppose is $<1$. Lemmas 1 and 2 are valid with the obvious changes (for example, $R(m, n)$ must be replaced by $\theta^{m}$ ). Lemma 3 holds as before, and the definition of canonical is the same.

We do not need any analogue of the conditions (5) or of Theorem 3 in the proof. We obtain an analogue of Lemma 4 by assuming the hypotheses of that lemma and using Theorem 2 with $n=2, \sigma_{1}=\theta^{\prime} / \theta^{\prime \prime}, \sigma_{2}=b_{22} / b_{21}$ and $p_{2}=-1$. We obtain

$$
\left|x_{1} x_{2}\left(x_{0}+\alpha x_{1}+\beta x_{2}\right)\right| \geqslant c_{23} /|m|^{c 24 \log d} \text {. }
$$

We divide the solutions of (10) into classes as before; then analogues of Lemmas 5 and 6 are easily obtained. This completes the proof of the nontotally real case of Theorem 1 .

\section{REFERENCES}

1. A. Baker, Effective methods in Diophantine problems, Proc. Sympos. Pure Math., vol. 20, Amer. Math. Soc., Providence, R.I., 1971, pp. 195-205. MR 47 \#324.

2. A sharpening of the bounds for linear forms in logarithms. I, II, Acta Arith. 21 (1972), 117-129; ibid, 24 (1973), 33-36.

3. - Effective methods in Diophantine problems. II, Proc. Sympos. Pure Math., vol. 24, Amer. Math. Soc., Providence, R. I., 1973, pp. 1-7. MR 49 \#2571.

4. Z. I. Borevič and I. R. Safarevič, Number theory, "Nauka", 1964; English transl., Pure and Appl. Math., vol. 20, Academic Press, New York, 1966. MR 30 \#1080; 33 \#4001.

5. J.W.S. Cassels, An introduction to Diophantine approximation, Cambridge Tracts in Math. and Math. Phys., no. 45, Cambridge Univ. Press, New York, 1957. MR 19, 396.

6. J.W.S. Cassels and H.P. F. Swinnerton-Dyer, On the product of three homogeneous linear forms and indefinite ternary quadratic forms, Philos. Trans. Roy. Soc. London Ser. A 248 (1955), 73-96. MR 17, 14.

7. T.W. Cusick, Diophantine approximation of linear forms over an algebraic number field, Mathematika 20 (1973), 16-23. MR 49 \#4942.

8. P. X. Gallagher, Metric simultaneous Diophantine approximation, J. London Math. Soc. 37 (1962), 387-390. MR 28 \#1167.

9. K. Mahler, Ein Übertragungsprinzip für lineare Ungleichungen, Časopis Pěst. Math. Fys. 68 (1939), 85-92. MR 1, 202. 
10. L. G. Peck, Simultaneous rational approximations to algebraic numbers, Bull. Amer. Math. Soc. 67 (1961), 197-201. MR 23 \#A111.

11. K. F. Roth, Rational approximations to algebraic numbers, Mathematika 2 (1955), 1-20; corrigendum, 168. MR 17, 242.

12. W. M. Schmidt, Simultaneous approximation to algebraic numbers by rationals, Acta Math. 125 (1970), 189-201. MR 42 \#3028.

13. D. C. Spencer, The lattice points of tetrahedra, J. Math. Phys. Mass. Inst. Tech. 21 (1942), 189-197. MR 4, 190.

DEPARTMENT OF MATHEMATICS, UNIVERSITY OF ILLINOIS, URBANA, ILLINOIS 61801

Current address: Department of Mathematics, State University of New York at Buffalo, Amherst, New York 14226 